\title{
JUMP HEIGHT ESTIMATION USING A SINGLE WEARABLE INERTIAL SENSOR MOUNTED ON SACRUM
}

\author{
Ramin Fathian ${ }^{1}$, Aminreza Khandan ${ }^{1}$, Loren Z.F. Chiu ${ }^{2}$, Hossein Rouhani ${ }^{1}$ \\ ${ }^{1}$ Mechanical Engineering Department, University of Alberta, Edmonton, AB, Canada \\ ${ }^{2}$ Faculty of Kinesiology, Sport, and Recreation, University of Alberta, Edmonton, AB, Canada
}

\begin{abstract}
Vertical jump performance assessment has been used as a tool to study and monitor physical performance in both athletic and non-athletic populations. One of the key parameters describing vertical jump performance is jump height. Although various methods are available for measuring the height, force platforms (FP) are the most precise, accurate, and reliable instrument, usually taken as the gold standard method. As force platforms are expensive and may not be practical outside of the lab environment, using wearable technologies like tri-axial inertial sensors (IS) is an inexpensive and reliable alternative. The objective of this study is to examine the technical validity and ability of using a sacrum mounted IS to estimate jump height. For this purpose, seven participants (age $26.3 \pm 2.0$ years) were recruited. Each participant was asked to perform two standing countermovement vertical jumps without arm swing on a FP (AMTI, USA. Sampling frequency $=2000 \mathrm{~Hz}$ ) while a tri-axial measurement sensor (Xsens, Netherland, Sampling Frequency $=100 \mathrm{~Hz}$ ) was placed on the sacrum. Standing countermovement jump is a widely used type of jump since it is easy to perform and closely related to movements in sports. The flight time was measured by finding the take-off and landing instants detected by analyzing the local peaks in the free vertical acceleration signal. Then, using the flight time measured by a single IS, the jump heights were estimated. Additionally, the jump heights for all the recorded jumps were calculated by implementing the take-off velocity method using the vertical component of the ground reaction force measured by the FP. Strong linear correlations were found between flight time found by FP and IS $\left(\mathrm{R}^{2}=0.76, \mathrm{~S}=0 \pm 1 \mathrm{Sec}\right.$ ), take-off velocity calculated by FP and flight time found by IS $\left(\mathrm{R}^{2}=0.80, \mathrm{~S}=0.017 \pm 0.005 \mathrm{~m} / \mathrm{s}\right)$, and jump height estimated using FP and IS $\left(\mathrm{R}^{2}=\right.$ $0.79, \mathrm{~S}=0.05 \pm 0.01 \mathrm{~m}$ ). Although the accuracy of this method, which employs the single wearable IS mounted on the sacrum, is sensitive to the take-off and landing time, there is error in jump height estimation $(2 \mathrm{~cm} \pm 1)$. This study demonstrates the accuracy of using a single IS in comparison to a FP to estimate standing countermovement jump height. Further investigations on a larger study sample are needed, however, wearable IS have the potential to accurately assess the physical performance of individuals in a rapid and portable manner.
\end{abstract}

